\title{
Riesgo de caries y su relación con las características clínicas bucales, sistémicas y conductuales de pacientes infantes
}

\author{
Caries risk and its relationship with clinic, systemic and behavioral features of infant patients \\ Stephanie Díaz Huamán ${ }^{l, a}$, Rosa Ana Melgar Hermoza $a^{2, b, c, d}$
}

\section{RESUMEN}

Objetivos: Determinar las características clínicas, sistémicas y conductuales; y su relación con el riesgo de caries de pacientes infantes atendidos por estudiantes de 5to. año de la Facultad de Estomatología, Universidad Peruana Cayetano Heredia, de enero del 2009 a abril del 2013. Material y métodos: Fue un estudio de corte transversal retrospectivo. La recolección de datos se realizó de las historias clínicas de pacientes infantes de 0 a 36 meses de edad. Se obtuvieron datos de: riesgo de caries, enfermedad sistémica, alteración de la estructura dental, frecuencia de higiene, frecuencia de consumo de azúcares extrínsecos, transmisión vertical, lactancia materna, lactancia artificial con edulcorante y amamantamiento nocturno. Se revisaron 170 historias clínicas de pacientes de 0 a 36 meses de edad. Resultados: Se encontró riesgo de caries alto en el 54,12\% de ellos, riesgo de caries moderado en el 22,35\% y riesgo de caries bajo en el 22,53\%. Conclusiones: Existió relación estadísticamente significativa entre riesgo de caries dental y los factores: frecuencia de higiene bucal, frecuencia de consumo de azúcares extrínsecos, amamantamiento nocturno y transmisión bacteriana.

PALABRAS CLAVE: Caries de infancia temprana, riesgo de caries, factores de riesgo. (Fuente: DeCS BIREME)

\section{SUMMARY}

Objectives: To determinate the clinical, systemic and behavioral features and its relationship with the caries risk of toddlers that were patients of the fifth year students of the School of Dentistry at Universidad Peruana Cayetano Heredia from January 2009 to April 2013. Methods: This was a transversal retrospective study. The

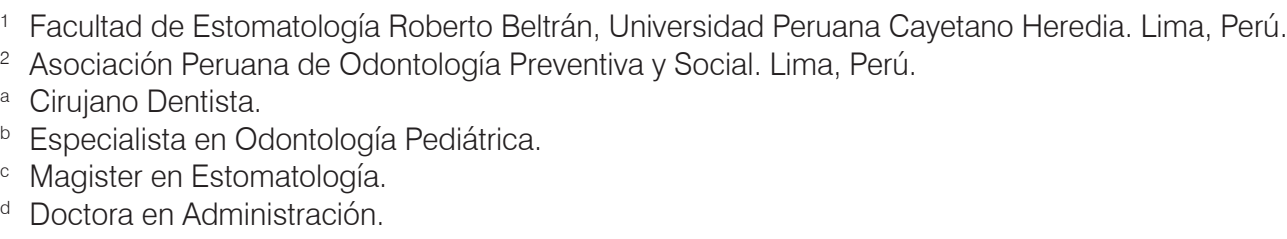


data was collected from the dental history of 0-36 months-old patients. We collected information such as caries risk, systemic diseases, dental structure alterations, extrinsic sugar intakes, vertical transmission, lactation, bottle-feeding with sweeteners and night breast-feeding. 170 dental histories were analyzed. Results: found that 54,12\% had high caries risk, 22,53\% had moderate caries risk and 22,35\% had low caries risk. Conclusions: there were significant relationship between the caries risk and some factors such as hygiene frequency, extrinsic sugar intakes, night breast-feeding and vertical transmission.

KEYWORDS: Early childhood caries, caries risk, risk factors. (Source: MeSH NLM)

\section{INTRODUCCIÓN}

En los últimos años se han estudiado varios factores reconociendo la naturaleza multifactorial de la caries dental, por lo que surge la necesidad de evaluar el riesgo de desarrollar Caries de Infancia Temprana (CIT) considerando las características particulares de los pacientes infantes. Pero, cada investigador y cada escuela evalúa sus propios factores de riesgo, existiendo una variada gama de posibilidades (1).

En infantes los factores etiológicos de la caries dental son mayormente controlados por los padres o tutores; es por esto que, es de gran importancia educarlos en el control de dichos factores. Una dieta rica en carbohidratos, la presencia de microorganismos cariogénicos, la falta de higiene bucal, el estado sistémico y la situación socioeconómica son factores influyentes en el desarrollo de CIT (2).

Existe un desconocimiento generalizado entre los padres sobre cuándo iniciar el cuidado bucal de sus niños, cuáles son los implementos necesarios para la higiene bucal y cómo hacerlo. La mayoría de adultos encuentra a la higiene bucal del niño, como una tarea incómoda e innecesaria. Es por ello que comenzar el cepillado de los niños es una situación frustrante para los padres, aplazándola por lo general el mayor tiempo posible. Cuando los niños comienzan a tener manifestaciones bucales irreversibles, tales como: lesiones de caries dentales amplias, infecciones pulpares, dolor o incluso infecciones de origen odontogénico; recién los padres buscan atención odontológica (3).

La alimentación del niño durante los 5 primeros años es fundamental para su desarrollo físico $\mathrm{y}$ mental, así como para su desarrollo psicomotor; por lo que requieren mayor atención. Por esta razón, es importante la presencia de vitaminas y proteínas en la dieta diaria. En el intento de los padres o tutores, que el infante se alimente a gusto, se tiende a aumentar la cantidad de azúcares en las comidas. Así, biberones con leche azucarada o bebidas con exceso de sacarosa en su composición son comunes en el día a día de los niños, por lo que es necesario controlar estos alimentos mediante la educación a padres de familia. Además, es necesario controlar al infante durante la etapa de ablactancia en la cual recibe los primeros alimentos alternos a la leche materna (a partir de los 6 meses). Así, el paciente corre riesgo de recibir alimentos azucarados innecesariamente como papillas de frutas con edulcorantes o pre envasados (4).

Los microorganismos presentes en la flora bucal del individuo como el Streptococcus mutans y Lactobacillus, disminuyen el $\mathrm{pH}$ bucal mediante la eliminación de ácidos, favoreciendo su multiplicación y la desmineralización de las piezas dentarias. El control de $\mathrm{pH}$ en pacientes infantes se realiza de dos maneras: evitando la colonización bacteriana en un ambiente nuevo y evitando que las bacterias realicen actividades reproductoras y metabolizadoras (5). La presencia de microorganismos cariogénicos y un ambiente rico en carbohidratos favorecen el crecimiento bacteriano aumentando el riesgo a tener Caries de Infancia Temprana $(6,7)$.

El objetivo del presente trabajo fue investigar si el riesgo de caries está asociado a las características clínicas, sistémicas y conductuales de los pacientes infantes atendidos por los alumnos del 5to. año del curso Clínica Integral Pediátrica V (CIP V) de la Facultad de Estomatología Roberto Beltrán de la Universidad Peruana Cayetano Heredia, durante el periodo: enero del 2009 a abril del 2013.

\section{MATERIALES Y MÉTODOS}

El presente trabajo fue de tipo transversal retrospectivo y fueron consideradas las historias clínicas de pacientes infantes de 0 a 36 meses de edad, desde enero del 2009 a abril del 2013 con los datos correctamente 
registrados. Se obtuvo el permiso respectivo del Departamento Académico responsable de la atención de los niños para la revisión de historias clínicas; y se aseguró la confidencialidad de las identidades del niño y operador.

Fueron estudiadas 170 historias clínicas que cumplieron con los criterios de inclusión. Los datos considerados para la investigación fueron: edad del infante, sexo, factores de riesgo de caries, enfermedades sistémicas, alteración de la estructura dental, frecuencia de higiene bucal, frecuencia de consumo de azúcares extrínsecos (FCAE), transmisión bacteriana vertical, lactancia materna, lactancia artificial con edulcorante y amamantamiento nocturno; datos que fueron registrados durante la consulta.

Todos los antecedentes fueron anotados durante la primera cita con el operador, como parte de la historia clínica, por lo que los datos de interés para el presente estudio fueron registrados en una ficha $a d h o c$, para el análisis correspondiente.

El riesgo de caries fue considerado como variable dependiente y la presencia de enfermedad sistémica, presencia de alteración de la estructura dental, frecuencia de higiene bucal, FCAE, transmisión vertical, tipo de lactancia y amamantamiento nocturno como variables independientes.

Los datos fueron analizados en el programa IBM SPSS Stadistics 21.0 utilizando el análisis bivariado, usando la prueba Chi Cuadrado. Los datos de prevalencia de edad, sexo y riesgo de caries fueron correlacionados con cada variable mencionada. Se realizó el análisis a partir de las tablas de contingencia y de los gráficos elaborados.

\section{RESULTADOS}

El promedio de edad fue 17 meses. Las niñas fueron el 51,75\% (88 niñas) y los varones el 48,25\%, (82 niños), primando los niños entre 8 y 14 meses de edad con $25,88 \%$ (44 infantes) y sólo el $12,36 \%$ (21 infantes) tenían de 29 a 36 meses de edad. La mayoría de los niños de la muestra tuvieron un riesgo de caries alto, siendo éste el 54,12\% (92 infantes); el $22,35 \%$ tuvieron riesgo de caries moderado y $22,53 \%$ un riesgo de caries bajo.
Se encontraron 3 infantes con enfermedad sistémica y con riesgo de caries alto; y 4 infantes con alteraciones de la estructura dental y con riesgo de caries alto también. No se encontraron diferencias significativas en la prueba estadística.

Al observar la frecuencia de higiene bucal, se encontró que entre los pacientes con riesgo de caries alto predominó la mala higiene bucal con 38,23\% (65 infantes), en la prueba estadística se encontró una relación altamente significativa $(\mathrm{p}=0,014)$, al igual que en la relación con la FCAE en donde también hubo una relación significativa $(\mathrm{p}=0,011)$.

Al analizar la presencia o ausencia de transmisión vertical, destaca que en el 74,11\% (126 infantes) existió signos de transmisión vertical y a la prueba estadística se concluyó que fue una relación altamente significativa $(\mathrm{p}=0,000)$.

Respecto al tipo de lactancia, la lactancia mixta predominó en el 39,41\% (67 infantes). Además, la mayoría de los pacientes con riesgo de caries alto también tenían lactancia mixta, más no hubo diferencia significativa $(p=0,787)$.

En la relación riesgo de caries y uso de edulcorantes durante la lactancia artificial, encontramos que hubo un mayor uso de éstos entre los pacientes con riesgo de caries alto: $13,53 \%$ (23 infantes) y se encontró una tendencia a ser estadísticamente significativo $(\mathrm{p}=$ $0,064)$. Finalmente, al relacionar riesgo de caries con la presencia o ausencia de amamantamiento nocturno, se encontró que fue mayor entre los pacientes de riesgo de caries alto con $50,59 \%$ ( 86 infantes) y menor entre los pacientes de riesgo de caries moderado con $12,36 \%$ (21 infantes). Al aplicar la prueba estadística se define que la relación fue altamente significativa $(\mathrm{p}$ $=0,000)($ Tabla 1).

Hubo menor frecuencia de higiene bucal en el $30,58 \%$ de los niños (52 infantes) y ésta fue menor en pacientes del sexo femenino en las niñas de 0 a 7 meses de edad, con 6,47\% (11 infantes) y mayor en pacientes femeninos de 22 a 28 meses con $6,47 \%$ (11 infantes). La relación entre edad y frecuencia de higiene bucal fue altamente significativa $(p=0,003)$ (Gráfico 1).

Observamos un mayor uso de edulcorante entre los pacientes con 29 a 36 meses y de 22 a 28 meses 
Tabla 1. Riesgo de Caries y variables de exposición en infantes atendidos.

\begin{tabular}{|c|c|c|c|c|c|c|c|c|c|}
\hline \multirow[b]{3}{*}{ Variable } & \multicolumn{6}{|c|}{ Riesgo de caries } & \multirow[b]{3}{*}{$\mathbf{x}^{2}$} & \multirow[b]{3}{*}{ gl } & \multirow[b]{3}{*}{$\mathbf{p}$} \\
\hline & \multicolumn{2}{|c|}{ Alto } & \multicolumn{2}{|c|}{ Moderado } & \multicolumn{2}{|c|}{ Bajo } & & & \\
\hline & $\mathbf{N}$ & $\%$ & $\mathbf{N}$ & $\%$ & $\mathbf{N}$ & $\%$ & & & \\
\hline \multicolumn{10}{|c|}{ Enfermedad sistémica } \\
\hline SI & 3 & 1,76 & 0 & 0 & 0 & 0 & 2,589 & 2 & 0,274 \\
\hline NO & 89 & 52,35 & 38 & 22,35 & 40 & 23,52 & & & \\
\hline \multicolumn{10}{|c|}{ Alteración de la estructura dental } \\
\hline SI & 4 & 2,35 & 0 & 0 & 0 & 0 & 3,473 & 2 & 0,176 \\
\hline NO & 88 & 51,76 & 38 & 22,35 & 40 & 23,52 & & & \\
\hline \multicolumn{10}{|c|}{ Frecuencia de higiene bucal } \\
\hline Buena $(\geq 2)$ & 27 & 15,88 & 19 & 11,18 & 21 & 12,35 & 8,556 & 2 & $0,014 *$ \\
\hline Mala $(<2)$ & 65 & 38,23 & 19 & 11,18 & 19 & 11,18 & & & \\
\hline \multicolumn{10}{|c|}{ Frecuencia de consumo de azúcares extrínsecos } \\
\hline $\operatorname{ALTO}(>6)$ & 13 & 7,65 & 12 & 7,05 & 3 & 1,76 & 9,011 & 2 & $0,011^{*}$ \\
\hline $\mathrm{BAJO}(\leq 6)$ & 79 & 46,47 & 26 & 15,29 & 37 & 21,76 & & & \\
\hline \multicolumn{10}{|c|}{ Transmisión vertical de microorganismos cariogénicos } \\
\hline SI & 73 & 42,94 & 33 & 19,41 & 20 & 11,76 & 16,648 & 2 & $0,000^{*}$ \\
\hline NO & 19 & 11,18 & 5 & 2,94 & 20 & 11,76 & & & \\
\hline \multicolumn{10}{|l|}{ Tipo de lactancia } \\
\hline Materna exclusiva & 23 & 13,53 & 11 & 6,47 & 13 & 7,65 & 1,721 & 4 & 0,787 \\
\hline Mixta & 67 & 39,41 & 27 & 15,88 & 26 & 15,29 & & & \\
\hline Artificial exclusiva & 2 & 1,18 & 0 & 0 & 1 & 0,59 & & & \\
\hline \multicolumn{10}{|l|}{ Uso de edulcorantes } \\
\hline SI & 23 & 13,53 & 9 & 5,29 & 3 & 1,76 & 5,509 & 2 & 0,064 \\
\hline $\mathrm{NO}$ & 69 & 40,59 & 29 & 17,06 & 37 & 21,77 & & & \\
\hline \multicolumn{10}{|c|}{ Amamantamiento nocturno } \\
\hline SI & 86 & 50,59 & 17 & 10,00 & 20 & 11,76 & 45,004 & 2 & $0,000^{*}$ \\
\hline NO & 6 & 3,53 & 21 & 12,36 & 20 & 11,76 & & & \\
\hline
\end{tabular}

*Asociación estadísticamente significativa (test Chi-Cuadrado) 


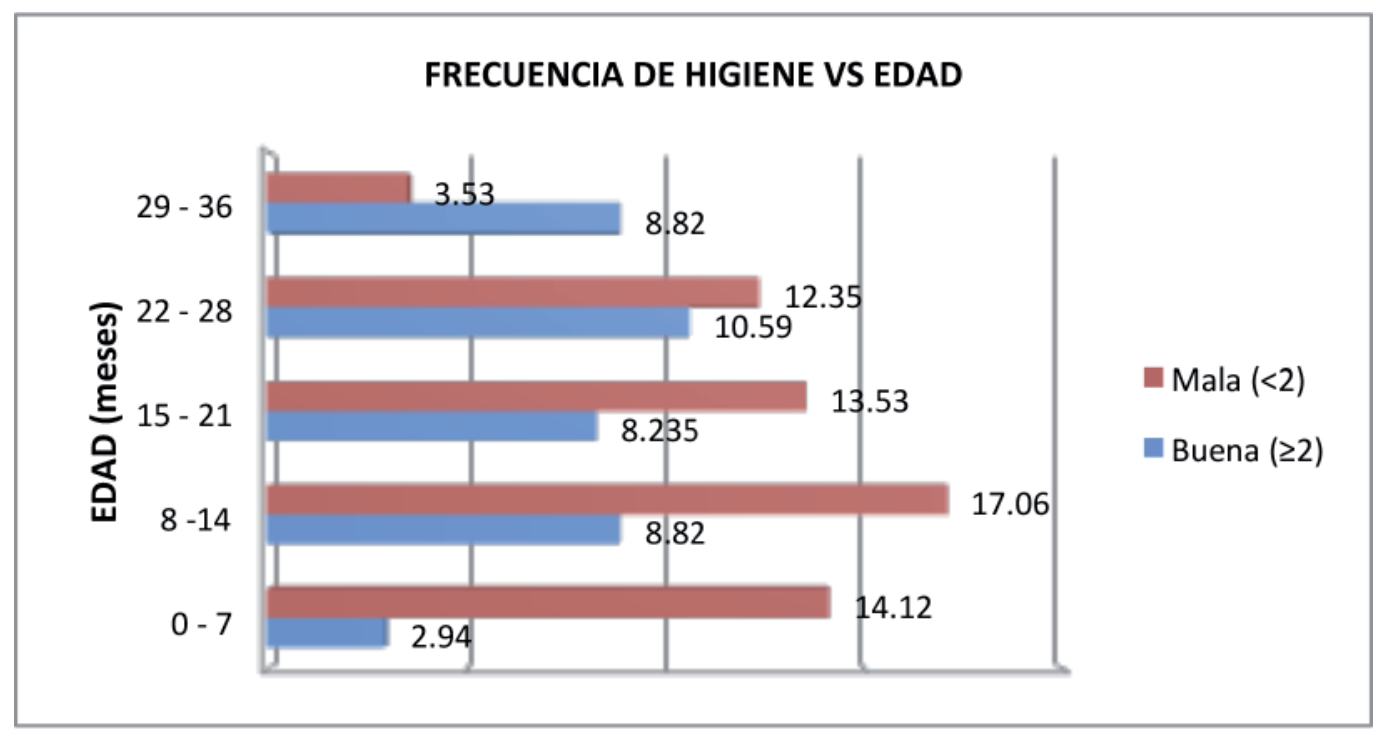

Gráfico 1. Relación entre edad y frecuencia de higiene bucal en infantes.

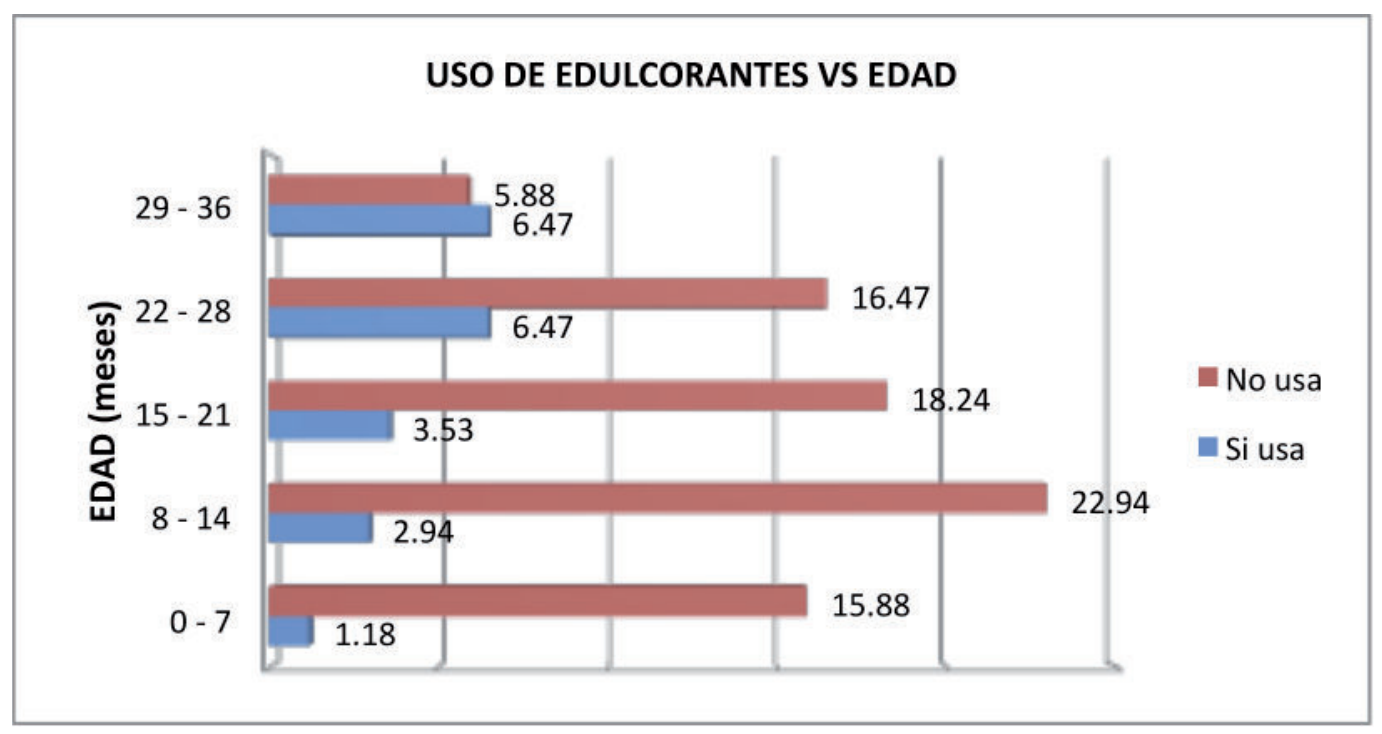

Gráfico 2. Relación entre la edad y el uso de edulcorantes durante la lactancia artificial en infantes.

de edad, ambos con $6,47 \%$ cada uno (11 infantes); y el menor uso se registró entre los pacientes de 0 a 7 meses de edad con 1,18\% ( 2 infantes). Al análisis estadístico se encontró que la edad y el uso de los edulcorantes tuvieron una diferencia altamente significativa $(p=0,000)$ y que el sexo y el uso de edulcorantes tuvieron una tendencia a ser significativa $(\mathrm{p}=0,064)($ Gráfico 2).
En el Gráfico 3, se destaca la presencia del amamantamiento nocturno entre las pacientes de sexo femenino, con $37,06 \%$ y los infantes de 8 a 14 meses con $21,18 \%$ (36 infantes). La lactancia durante la noche fue menor entre los pacientes de 29 a 36 meses con 5,88\% (10 infantes). Luego de efectuado el análisis estadístico concluimos que la relación entre sexo y amamantamiento nocturno no es significativa, más su relación con la edad sí es lo es $(\mathrm{p}=0,034)$. 


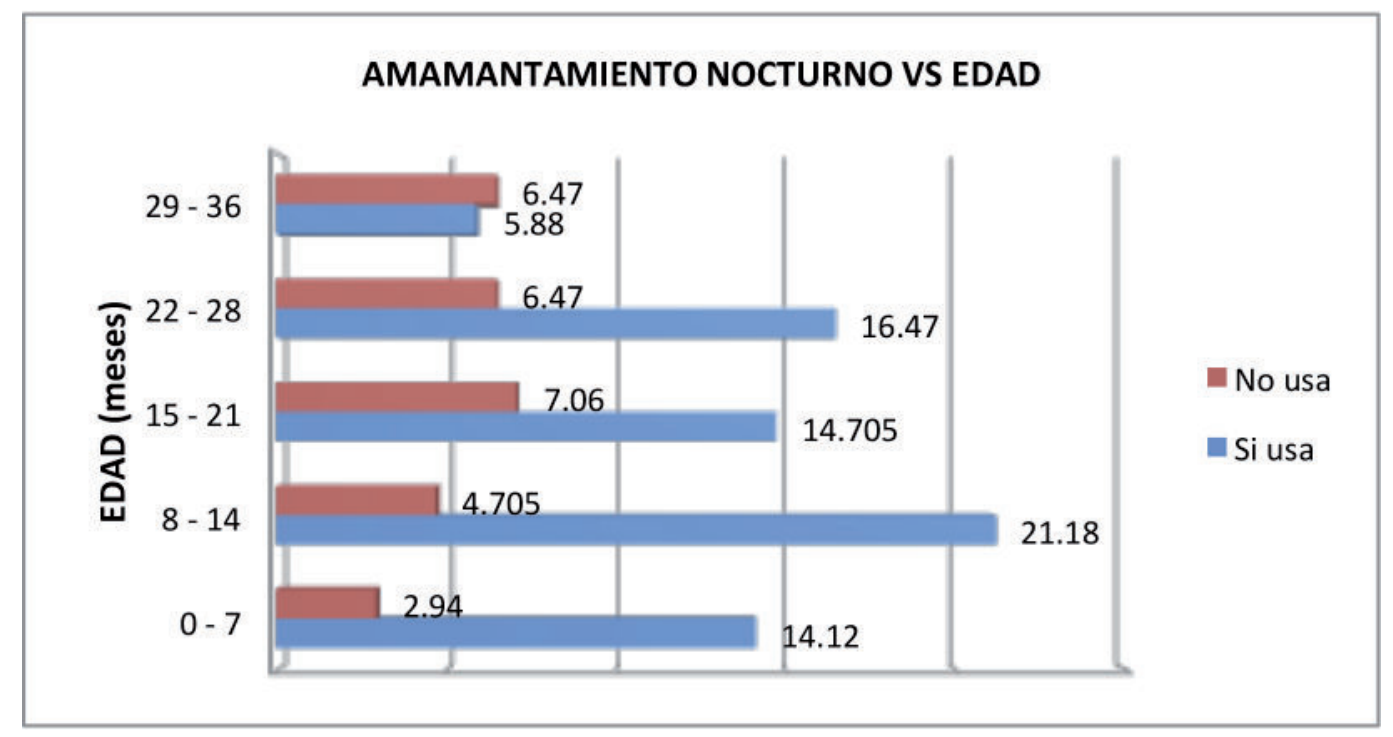

Gráfico 3. Relación entre edad y amamantamiento nocturno en infantes.

\section{DISCUSIÓN}

Más de la mitad de infantes tuvieron un alto riesgo de desarrollar caries dental hasta la edad de 3 años, seguida de la población con riesgo de caries moderado y una menor cifra para los pacientes con riesgo de caries bajo. Además, encontramos una mínima incidencia de alteraciones de estructura y de pacientes con enfermedades sistémicas, por lo que no se encontró diferencia significativa al relacionarla con el riesgo de caries. Probablemente, porque cuando los operadores de pregrado buscan pacientes, estos deben ser sistémicamente sanos. Es por ello, que la población del presente estudio presentó limitaciones en cuanto a variedad.

En esta investigación encontramos que la relación entre frecuencia de higiene bucal y riesgo de caries fue significativa $(\mathrm{p}=0,014)$. La relación entre transmisión vertical y riesgo de caries fue altamente significativa también $(\mathrm{p}=0,000)$. Cogulu y col. (8) encontraron que en pacientes inicialmente sanos, hubo un alto desarrollo de caries cuando había un alto índice de placa por una baja frecuencia de higiene bucal $(\mathrm{p}=0,017)$; este autor afirma en su estudio, que la presencia del Streptococcus mutans, por transmisión vertical, fue determinante para el desarrollo de caries dental $(\mathrm{p}=0,11)$.

Así mismo, existió una relación altamente significativa entre la edad de los infantes y frecuencia de higiene bucal $(\mathrm{p}=0,003)$. En los estudios de Quiñonez y col. (9) y Zaror y col. (10), se destaca el papel del cuidador en la salud bucal del niño; teniendo como resultado la estrecha relación entre edad e higiene bucal, manifestando su mejoría progresiva a medida que la edad del niño aumentaba.

En el presente estudio, se encontró que la relación entre riesgo de caries y FCAE fue significativa $(\mathrm{p}=0,011)$, al igual que en estudios clásicos de dieta-caries realizados por Stephan en 1944 (11) y Gustaffson y col. (12) en 1954, por los que sabemos que el $\mathrm{pH}$ bucal es afectado por los 'golpes' de glúcidos fermentables.

No se encontró relación significativa entre tipo de lactancia que recibió el niño y el riesgo de caries dental $(p=0,787)$. Al igual que en el estudio de Abanto y Jotta (13) requerimos de un grupo de estudio de mayor número y centrarnos en la lactancia como factor determinante en el desarrollo de lesiones de caries para tener resultados más detallados.

El $72,35 \%$ de la población estudiada lactaba durante la noche. La relación del riesgo de caries y el amamantamiento nocturno fue estadísticamente significativa $(p=0,000)$ y la edad y el amamantamiento nocturno tuvieron una relación estadísticamente significativa $(p=0,034)$. En el estudio de Hallet y Rourke (14) se encuentró que el alto índice de pacientes que lactaban en la noche influenciaba la 
aparición de CIT $(\mathrm{p}<0,005)$ y ésta era más frecuente entre los niños de menor edad que en los mayores.

La presente investigación demostró que los edulcorantes no fueron tan usados en la población estudiada, pero aún asi fue prevalente $(20,58 \%)$ y la relación con el riesgo de caries no fue estadísticamente significativa. En la presente investigacíon se encontró que la edad y el uso de edulcorantes tuvo una relación altamente significativa $(\mathrm{p}=0,000)$. En estudios como el de Da Silva y col. (15), se concluye que el uso temprano de azúcares aumenta el riesgo de caries y en el de Abanto y Jotta (13) se destaca que los niños de mayor edad tuvieron un contacto más frecuente con el azúcar.

Concluimos que entre riesgo de caries y los factores: frecuencia de higiene, FCAE, transmisión vertical y amamantamiento nocturno, tuvieron una relación estadísticamente significativa.

\section{Correspondencia:}

Stephanie Díaz Huamán

Av. Colombia N ${ }^{\circ}$ 171, Pueblo Libre. Lima, Perú .

Correo electrónico: Stephanie.Diaz@upch.pe

\section{REFERENCIAS BIBLIOGRÁFICAS}

1. Quiñonez R, Keels M, Vann W, Mclver F, Heller K, Whitt J. Early childhood caries: analysis of psychosocial and biological factors in a high-risk population. Caries Res. 2001; 35(5): 376-83.

2. Mattos M, Melgar R. Riesgo de caries dental. Rev Estomatol Herediana. 2004; 14(1): 101-106.

3. Kanashiro C, Medrano G. La enfermedad caries dental. En: Castillo R, Perona M, Kanashiro C, Perea M, Silva F. Estomatología Pediátrica. 1ra Edición. Lima: ed. Ripano; 2011. p. 100-10.

4. American Academy of Pediatric Dentistry. Policy on dietary recommendations for infants, children and adolescents. AAPD. 2012; 34(6): 56-8.
5. Birkeland J, Broch L, Jorkjend L. Caries experience as a predictor for caries incidence. Community Dent Oral Epidemiol. 1976; 4(2):66-9.

6. Berkowitz R, Amante A, Billing R. Dental caries recurrence following clinical treatment for severe early childhood caries. Pediatr Dent. 2011; 33(7):510-4.

7. Tanzer JM, Thompson A, Wen ZT, Burne A. Streptococcus mutans: fructose transport, xylitol resistance and virulence. J Dent Res. 2006; 85(4):36973.

8. Contreras N, Valdivieso M, Cabello E. Nivel de conocimientos y prácticas de medidas preventivas de profesionales de salud sobre caries dental en el infante. Rev Estomatol Herediana. 2008; 18(1):29-34.

9. Cogulu D, Ersin NK, Uzel A, Eronat N, Aksit S. A long-term effect of caries-related factors in initially caries-free children. Int J Paediatr Dent. 2008; 18(5):361-7.

10. Zaror S, Pineda T, Orellana J. Prevalencia de caries temprana de la infancia y sus factores asociados en niños chilenos de 2 y 4 años. Int J Odontostomat. 2011; 5(2):171- 7.

11. Stephan RM. Intra-oral hydrogen ion concentrations associated with dental caries activity. J Dent Res. 1944; 23 (4):257-66.

12. Gustafsson B, Quensel C, Lanke L, et al. The Vipeholm dental caries study; the effect of different levels of carbohydrate intake on caries activity in 436 individuals observed for five years. Acta Odontol Scand. 1954; 11(3-4): 232-364.

13. Abanto J, Jotta A. Patrones de lactancia en bebés, su primer contacto con el azúcar y el dentista. Acta Odont Venez. 2010; 48(2):1-11.

14. Hallet K, Rourke P. Social and behavioural determinants of early childhood caries. Aust Dent J. 2003; 48(1)2733.

15. Da Silva G, Costa B, Ribeiro M, Teixeira L. Breastfeeding and sugar intake in babies with cleft lip and palate. CPCJ 2003; 40 (1):84-7.

Recibido: 14/09/2013

Aceptado: 07/02/2014 